\title{
Pain-diminishing and quality of life-related outcomes of Kinesio taping in patients on non-steroidal anti-inflammatory drug therapy for post-thoracotomy pain syndrome
}

\author{
Hülya Kurt Saruhan (1), Murat Toprak (1) \\ Department of Physical Medicine and Rehabilitation, Yüzüncü Yll University Faculty of Medicine, Van, Turkey
}

Received: December 12, 2018 Accepted: August 19, 2019 Published online: May 18, 2020

\begin{abstract}
Objectives: This study aims to evaluate pain-diminishing and quality of life-related outcomes of Kinesio taping in patients on non-steroidal anti-inflammatory drug (NSAID) therapy for post-thoracotomy pain syndrome (PTPS).

Patients and methods: Between February 2016 and May 2017, a total 60 patients (39 males, 21 females; median age 43.5 years; range, 18 to 76 years) with PTPS were included in this single-center, prospective, randomized study. The patients were randomized into two groups based on five-day pain management protocol including NSAIDs per se (NSAID group; $n=30$ ) and NSAIDs plus Kinesio taping (NSAID-KT group; $n=30$ ) groups. Pain intensity (via visual pain scale [VAS]), neuropathic pain (Leeds Assessment of Neuropathic Symptoms and Signs [LANSS] Pain Scale), major chronic pain symptoms (via Short-Form McGill Pain Questionnaire [SF-MPQ-2]), and quality of life (via Nottingham Health Profile [NHP]) were assessed before and after five-day treatment period.

Results: In both NSAID and NSAID-KT groups, treatment was associated with a significant decrease in the VAS-pain (p<0.001), LANSS Pain Scale scores (from median 8.0 to 3.0 and from median 18.5 to 15.0 , respectively, $\mathrm{p}<0.001$ for each), SF-MPQ scores ( $\mathrm{p}<0.001$ ), and physical mobility and pain domains of NHS ( $\mathrm{p}<0.001$ for each).

Conclusion: In conclusion, our study findings indicate no additional benefit of KT application on further amelioration of long-term PTPS in patients under NSAID analgesia. Both NSAID and NSAID-KT treatments produced a significant improvement in the VAS, LANSS, SF-MPQ scores, and in the quality of life after five-day treatment.
\end{abstract}

Keywords: Kinesiotaping, long-term, non-steroidal anti-inflammatory drug, pain, post-thoracotomy pain syndrome, quality of life.

Post-thoracotomy pain syndrome (PTPS) is a significant and relatively common complication of thoracic surgery which affects 21 to $61 \%$ of patients and may persist up to four to five years after surgery in $30 \%$ of patients. ${ }^{[1-6]}$ While the exact pathogenesis still remains unclear, cumulative evidence indicates PTPS to be a combination of neuropathic and nonneuropathic (myofascial) pain. ${ }^{[4-8]}$

In current practice, pain control remains a challenge in the postoperative care in thoracic surgery which may necessitate the use of more than one form of therapy or complementary methods (i.e., acupuncture, transcutaneous electrical nerve stimulation, or physical therapy) to control pain and minimize disability. ${ }^{[6,9-16]}$

Kinesio taping (KT) is a method to support physiotherapy with application of an elastic tape to the skin which is specifically designed to facilitate the body's natural healing processes after trauma or inflammation by modifying the underlying soft tissue spaces. ${ }^{[17]}$ It is proposed to have several potential effects depending on the technique and degree of tape stretch. ${ }^{[17]}$ This involves alignment of the fascial tissues, creation of more space by lifting fascia and soft tissue above area of pain, sensory stimulation, and assisting in edema reduction by increasing the

Corresponding author: Hülya Kurt Saruhan, MD. Yüzüncü Yll Üniversitesi Tıp Fakültesi Fiziksel Tıp ve Rehabilitasyon Anabilim Dalı, 65080 Zeve, Van, Türkiye. e-mail: hulyakurt_89@icloud.com 
blood circulation and lymphatic drainage. ${ }^{[17-19]}$ In recent years, KT has become increasingly used as a method combined with other pharmacological and physiotherapeutic interventions in the management of sports injuries, postoperative complications, and pain problems including myofascial pain. ${ }^{[12,18,19]}$

The use of KT as a measure to alleviate postoperative pain has been addressed only in a few trials, which indicated its efficacy in orthopedic, gastrointestinal, and thoracic surgery patients during early postoperative rehabilitation. ${ }^{[12,13,18,20]}$ Hence, there is a need for high-quality controlled studies to confirm the potential efficacy of KT on postoperative pain management combined with more traditional antalgic therapies. ${ }^{[6,21]}$

To the best of our knowledge, there is no study to date which has addressed the utility of KT in patients with long-term PTPS. In the present study, therefore, we aimed to evaluate pain-diminishing and quality of life (QoL)-related outcomes of Kinesio taping in patients on NSAID therapy for PTPS.

\section{PATIENTS AND METHODS}

This single-center, prospective, randomized study was conducted at Yüzüncü Yll University Faculty of Medicine between February 2016 and May 2017. A total 60 patients ( 39 males, 21 females; median age 43.5 years; range, 18 to 76 years) with PTPS for at least two weeks after the operation were included. All patients were randomized using the random number tables into two groups based on five-day pain management protocol including NSAIDs per se (NSAID group; $\mathrm{n}=32$ ) and NSAIDs plus Kinesio taping (NSAID-KT group; $n=32$ ) groups. Exclusion criteria were as follows: pediatric age, pregnancy, renal or hepatic dysfunction, presence of infection or open wound within the region to be taped, post-thoracotomy pain for shorter than two weeks, hypersensitivity to KT or NSAIDs, and recent use (within 4 weeks prior to enrolment) of NSAIDs. Final study population subjected to analysis was composed of 30 patients in each group with the exclusion of two patients (due to gastrointestinal adverse events and lost-to-follow up) in the NSAID group and two patients (due to allergic reaction) in the NSAID-KT group during the study period.

A written informed consent was obtained from each patient. The study protocol was approved by Yüzüncü Yil University Faculty of Medicine Clinical Research Ethics Committee (Date: 28/02/2017; No. 01). The study was conducted in accordance with the principles of the Declaration of Helsinki.

\section{Data collection}

Data including patients' demographics (age and sex), anthropometrics, and duration of postthoracotomy pain were recorded in each patient at the study enrolment. Pain intensity (via visual pain scale [VAS]), neuropathic pain (Leeds Assessment of Neuropathic Symptoms and Signs [LANSS] Pain Scale), major chronic pain symptoms (via Shortform McGill Pain Questionnaire [SF-MPQ-2]), and QoL (via Nottingham Health Profile [NHP]) were assessed before and after five-day treatment period.
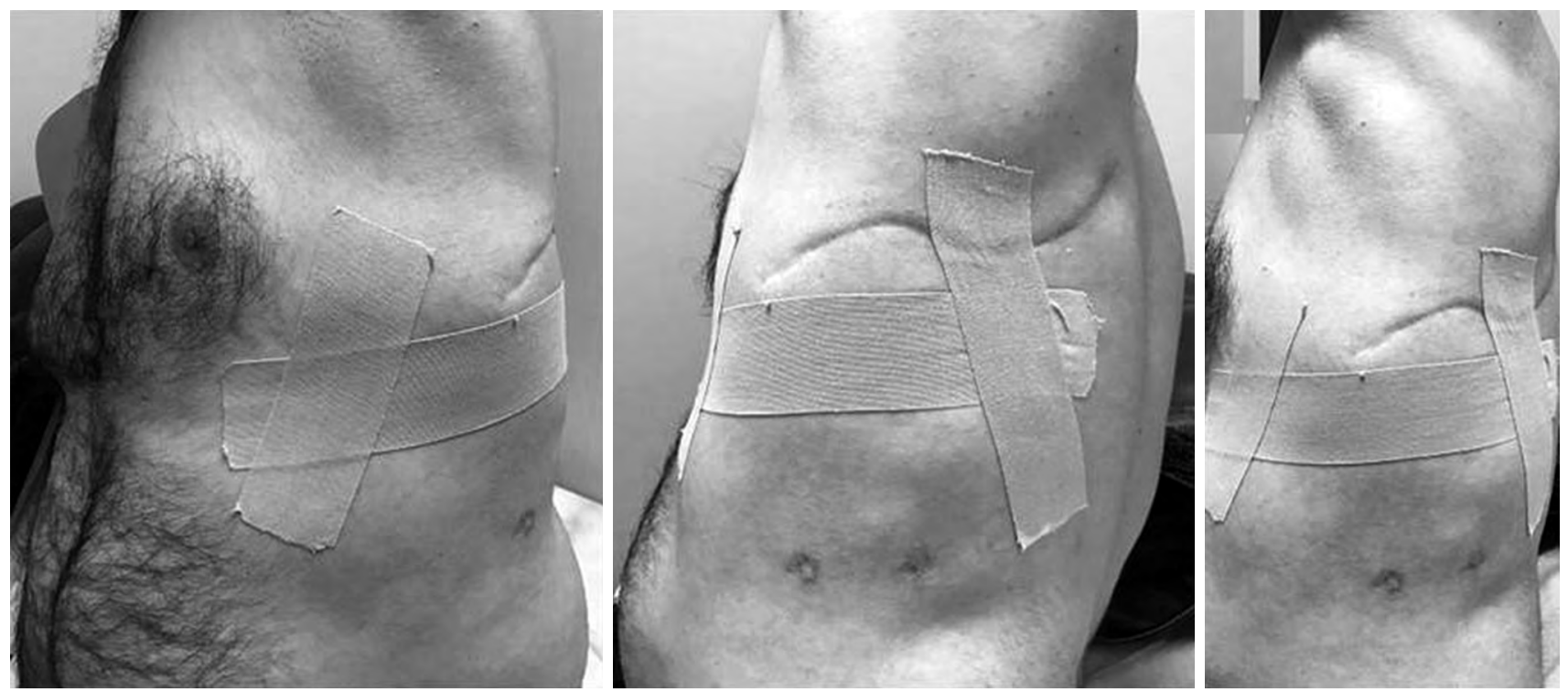

Figure 1. Kinesio taping application. 


\section{Kinesio taping}

In the NSAID-KT group, Kinesio Tex Gold ${ }^{\mathrm{TM}}$ (Kinesio Holding Corp., Albuquerque, NM, USA), a $100 \%$ cotton, latex-free, $5-\mathrm{cm} \times 0.5-\mathrm{mm}$ elastic tape was applied by a specialized physiotherapist to the chest skin in accordance with the principles and techniques described by Kase et al. ${ }^{[17]}$ Accordingly, KT was applied to the chest skin with two overlapping I-shape bands to decongest the main painful area by lifting up the underlying tissues and with $50 \%$ tension in the center of each strip. The I-shaped KT application was directly above the main pain trigger point (target area) identified by chest palpation (Figure 1). The patients received etodolac $(600 \mathrm{mg} /$ day $)$ as the analgesic agent.

\section{VAS-pain}

TheVAS-painis a self-administered, unidimensional measure of pain intensity, which has been widely used in diverse adult populations. It is a continuous $100-\mathrm{mm}$ scale anchored by two verbal descriptors for pain intensity, including "No pain" (score of 0) and "Worst imaginable pain" (score of 100 [100-mm scale]). It provides a range of scores from 0 to 100 with higher scores indicating greater pain intensity. ${ }^{[22,23]}$ In this study, the VAS-pain scores were assessed in different settings including resting, during deep breathing, coughing, and bending to the operated side.

\section{LANSS pain scale}

LANSS Pain Scale, developed by Bennett in 2001, ${ }^{[24]}$ provides immediate information on discrimination of neuropathic pain from nociceptive pain in the clinical setting. Total score of the scale is 24 with likelihood of neuropathic mechanisms indicated by scores $\geq 12$. The Turkish version of the LANSS Pain Scale has been validated by Yucel et al. ${ }^{[25]}$ in 2004.

\section{SF-MPQ}

The SF-MPQ, a shorter version of the MPQ developed by Melzack in $1987,{ }^{[26]}$ is an intervieweradministrated, multidimensional measure of perceived pain in adults with chronic pain. It is comprised of 15-item Pain Rating Index, one item for the present pain intensity, and one item for a 10-cm VAS for the average pain. ${ }^{[23,26]}$ The pain Rating Index has two subscales including sensory subscale with 11 words or items and affective subscale with four words or items, each selected word is rated on an intensity scale from 0 (none) to 3 (severe). The total Pain Rating Index score is obtained by summing the item scores (range 0-45) with higher scores indicating the greater the pain levels. Scores on the present pain intensity range from 0 to 5 , while those on the VAS from 0 to $10 .^{[23,26]}$ The Turkish version of the SA-MPQ was validated by Yakut et al. ${ }^{[27]}$ in 2007.

\section{NHP}

The NHP indicates the effect of ill-health on QoL. ${ }^{[28]}$ The 38 items in the NHP are divided into six domains: physical mobility (8 items), pain (8 items), sleep (5 items), fatigue (3 items), social isolation (5items), and emotional reactions ( 9 items). Each domain is independently scored from 0 to 100 with higher scores indicating a poor health function in a particular domain. The QoL of respondents is calculated by generalizing the scores in six domains. ${ }^{[28]}$ The Turkish version of the NHS was validated by Kucukdeveci et al. ${ }^{[29]}$ in 1997.

\section{Statistical analysis}

According to the post-hoc power analysis, a sample size of 30 in each group achieves $89 \%$ power to detect a difference of 3.5 between the null hypothesis mean of 18.5 and the alternative hypothesis mean of 15.0 with a known standard deviation of 6.0 and with a significance level (alpha) of 0.05 using a two-sided one-sample.

Statistical analysis was performed using the SPSS for Windows version 16.0 (SPSS Inc., Chicago, IL, USA). Descriptive data were expressed in median (min-max) and number and frequency. The Pearson chi-square test was used to analyze categorical data, while the Mann-Whitney $U$ and Wilcoxon tests were used to analyze numerical data between the treatment groups and change from baseline in each treatment group, respectively. The Power Analysis and Sample Size (PASS) software was used for power calculation using the LANSS Pain Scale scores as the variable. A $p$ value of $<0.05$ was considered statistically significant.

\section{RESULTS}

Compared to the NSAID group, in the NSAID-KT group, the number of younger patients was significantly higher $(\mathrm{p}=0.012)$ and the number of patients with a longer ( $>8$ weeks) duration of PTPS was significantly higher $(63.3 \%$ vs. $36.7 \%, \mathrm{p}=0.020)$ (Table 1$)$.

In both NSAID and NSAID-KT groups, treatment was associated with a significant decrease in the VAS pain scores for all tested conditions $(p<0.001$ for each), with a significant decrease in the LANSS Pain Scale scores (from median 8.0 to 3.0 and from median 18.5 to 15.0 , respectively, $\mathrm{p}<0.001$ for each) and with a significant decrease in all subdomains of SF-MPQ 


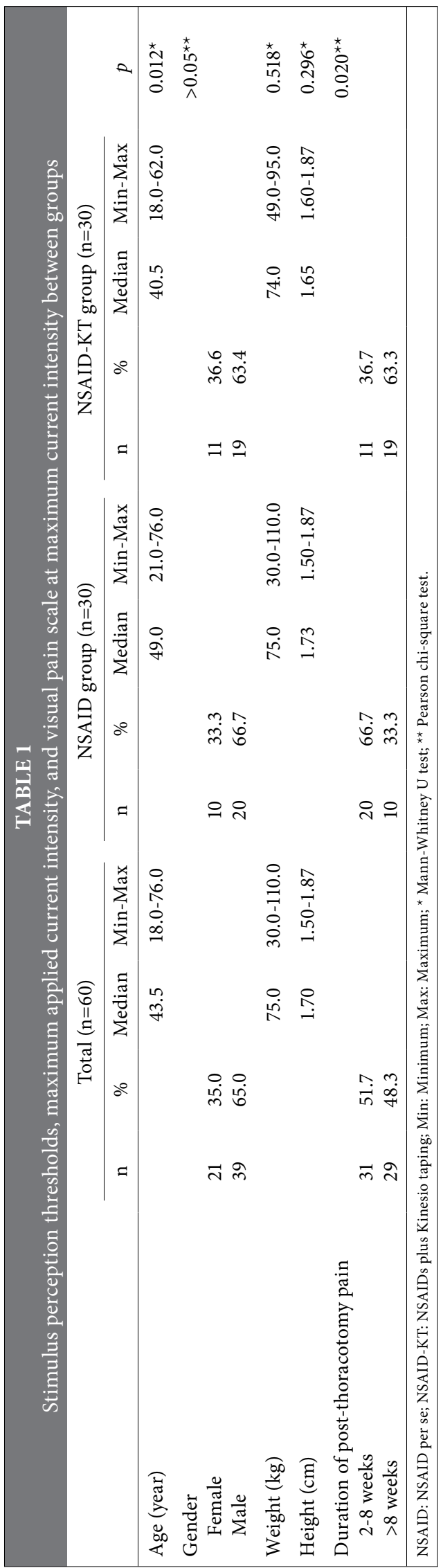

$(\mathrm{p}<0.001$ for each) (Table 2). In the pre-treatment period, the LANSS Pain Scale scores were $>12$ among $22(73.3 \%)$ patients in the NSAID-KT group and in eight (26.7\%) patients in the NSAID group. The NHS scores significantly decreased for physical mobility $(\mathrm{p}<0.001)$, pain $(\mathrm{p}<0.001)$, sleep $(\mathrm{p}<0.05)$ and fatigue $(\mathrm{p}<0.05)$ domains in the NSAID-KT group, but only for physical mobility and pain domains in the NSAID group ( $\mathrm{p}<0.001$ for each) (Table 2 ).

In the pre-treatment period, the LANSS Pain Scale scores $(p=0.001)$ and SF-MPQ scores for affective subdomain ( $\mathrm{p}=0.001)$, present pain intensity $(\mathrm{p}<0.001)$, and VAS for pain $(\mathrm{p}=0.010)$ were significantly higher in the NSAID-KT group than in the NSAID group (Table 2). After five-day treatment, the LANSS Pain Scale scores $(\mathrm{p}=0.002)$, SF-MPQ scores for affective subdomain $(\mathrm{p}=0.001)$, and present pain intensity scores $(\mathrm{p}=0.049)$ remained significantly higher in the NSAID-KT compared to the NSAID group, whereas VAS pain scores related to the SA-MPQ were similar after the treatment between NSAID and NSAID-KT groups, as were the VAS pain scores and NHP scores (apart from significantly lower values for physical mobility in NSAID-KT group, $\mathrm{p}=0.039$ ) (Table 2).

\section{DISCUSSION}

In the present study, we found that five-day NSAID therapy and NSAID plus KT protocol yielded similar efficacy on pain reduction in patients with PTPS (persistent for at least two weeks after the operation). The VAS-pain (for all conditions tested), SF-MPQ (for all domains), and LANSS Pain Scale scores were significantly improved in both groups. However, the impact of treatments on QoL seems to differ with respect to certain domains of NHL with significantly improved physical mobility and pain scores in both treatment groups, whereas improvement in only sleep and fatigue scores was seen in the NSAID-KT group.

Efficacy of KT in postoperative pain reduction was evaluated in a previous study including 92 patients undergoing lobectomy for lung cancer who were randomized to $\mathrm{KT}$ group (standard postoperative analgesia plus KT) or placebo-control group (standard postoperative analgesia plus usual dressing tapemimicking KT). ${ }^{[12]}$ The authors found a greater postoperative pain reduction from Day 1 to Day 5 in the KT group than in controls (VAS -3 vs. -2), as well as significantly lower median VAS scores and less frequent persistence of moderate-to-severe intensity chest pain (VAS $\geq 3$ ) in the KT treatment group (7\% vs. $24 \%)$ at 30 days after lobectomy. In addition, 


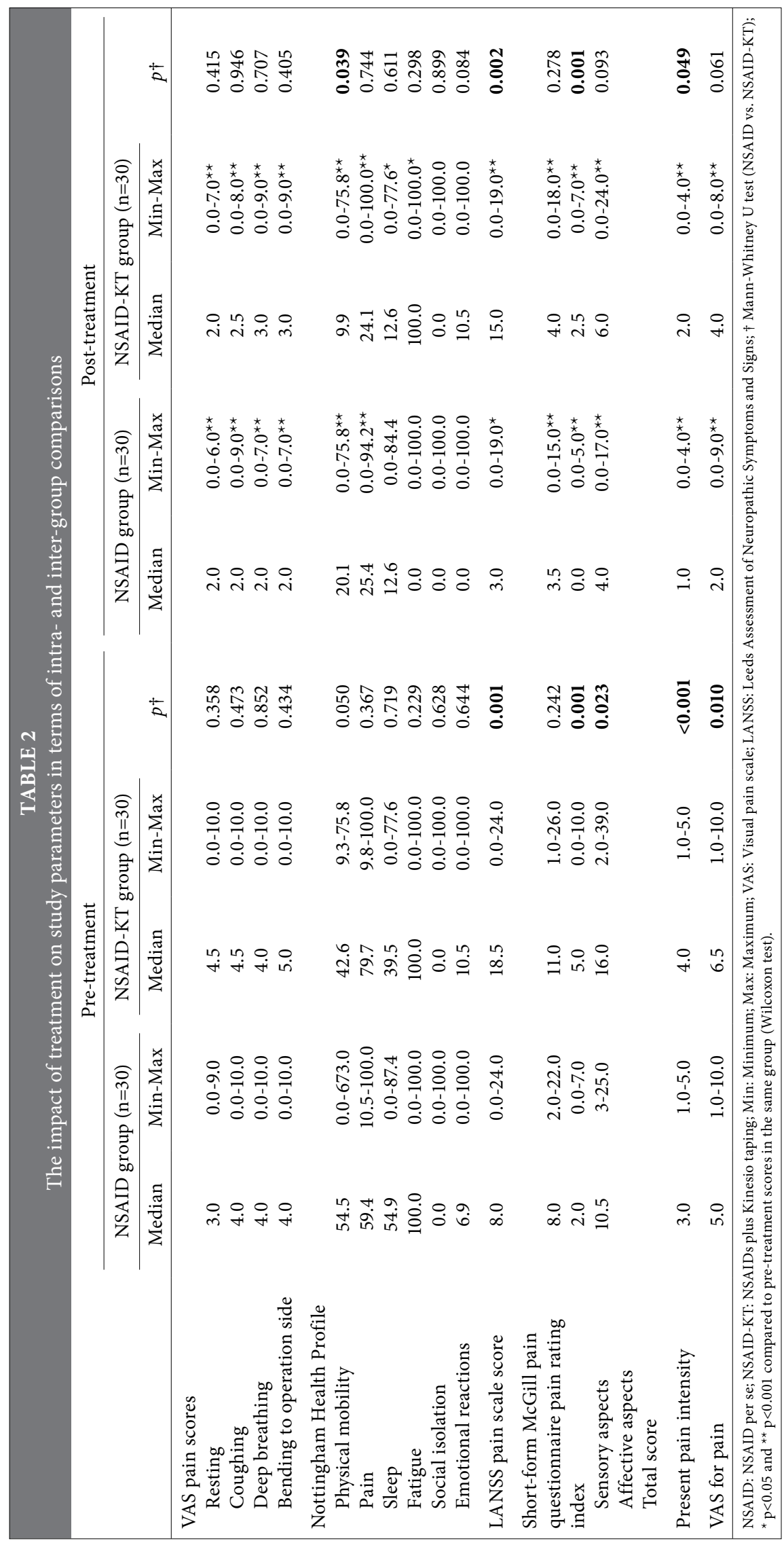


the increased efficacy of KT on postoperative pain control was observed, when the patients discontinued intravenous/epidural analgesia and switched to less effective oral medications. Also, the observed outcome of one-point less VAS pain score in the $\mathrm{KT}$ group might be considered a small difference in a subjective reporting, while this enabled lesser need for supplemental analgesia and lower rate of moderate-tosevere chest pain (VAS $\geq 3$ ) at 30 days after lobectomy in the KT group. ${ }^{[12]}$

Our findings revealed a significant decrease in the VAS pain scores for all conditions tested after treatment in both NSAID (from 4.0-4.6 to 1.9-2.2) and NSAID-KT (from 4.5-5.0 to 2.6-2.7) treatment groups. This seems notable given the concomitantly improved QoL scores in physical mobility and pain domains in both groups. Hence, our findings support the consideration of one-point reduction in VAS scores to have a positive impact on patient QoL via decreased inhibition of painful movements and reduced need for additional pain killers. ${ }^{[12,30]}$ However, given that no superiority of NSAID-KT over NSAID was identified in terms of reduction in VAS pain scores, our findings seem to support the consideration of VAS score 3 to be the threshold of postoperative pain above which $\mathrm{KT}$ is ineffective as an auxiliary analgesic measure. ${ }^{[12]}$

In a prospective, randomized-controlled study in 39 patients investigating the impact of KT on postoperative morbidity after median sternotomy, KT was considered to be a low-risk, non-pharmacological, cost effective, and promising method for control of early postoperative pain after median sternotomy. ${ }^{[13]}$ Since acute postoperative pain is considered amongst the strongest predictors of PTPS, ${ }^{[5,31]}$ the potential role of aggressive perioperative anesthetic and analgesic techniques has been suggested in reducing the incidence of PTPS. ${ }^{[32]}$

A possible long-term effect of $\mathrm{KT}$, albeit removed at hospital discharge, was reported on reduced late postoperative chest pain on postoperative Day 30 among lobectomy patients. ${ }^{[12]}$ Thus, adopting an aggressive multimodal perioperative pain management regimen and application of KT in the early postoperative period is considered likely to offer a favorable late effect to prevent PTPS..$^{[6,12,33]}$ On the other hand, our findings seem not to indicate KT application as an effective auxiliary technique to ongoing NSAID analgesia in further reduction of long-term/chronic PTPS, although there is a possibility of better improvement in sleep and fatigue-related QoL scores with the application of KT.
Nonetheless, there are some limitations to this study. First, relatively low sample size might prevent us to achieve the statistical significance concerning the KT application in terms of pain-related parameters as well as to generalize our findings to the entire population. Second, while the duration of pain and patient age differed significantly at baseline between the treatment groups, no significant difference was observed in the LANSS scores according to duration of pain (2-12 weeks vs. $>12$ weeks) before and after treatment in both KT and NSAID groups. Hence, alongside the likelihood of age-independent variability of postoperative pain previously reported in the literature, ${ }^{[34]}$ these baseline differences seem not to refer to a significant source of bias for the current study. Third, the lack of a placebo/sham group as well as lack of data on long-term efficacy is another limitation which, otherwise, would extend the knowledge achieved in the current study. However, all thoracotomy operations were performed in a single institution by members of the same surgical team, and randomization and tape application were performed by a single specialized physiotherapist which revealed a homogeneous and unbiased management.

In conclusion, our findings indicate no additional benefit of KT application on further amelioration of long-term post thoracotomy pain in patients under NSAID analgesia. In the present study, both NSAID and NSAID-KT treatments revealed a significant improvement in VAS, LANSS, and SF-MPQ scores as well as in the QoL after five-day treatment. Future large-scale, prospective, randomizedcontrolled studies are needed to address the efficacy of KT application in the early postoperative period in prevention of PTPS and its efficacy in amelioration of persistent long-term post-thoracic pain, particularly in combination with oral analgesics effective on neuropathic component of pain.

\section{Declaration of conflicting interests}

The authors declared no conflicts of interest with respect to the authorship and/or publication of this article.

\section{Funding}

The authors received no financial support for the research and/or authorship of this article.

\section{REFERENCES}

1. Dajczman E, Gordon A, Kreisman H, Wolkove N. Longterm postthoracotomy pain. Chest 1991;99:270-4.

2. Perttunen K, Tasmuth T, Kalso E. Chronic pain after thoracic surgery: a follow-up study. Acta Anaesthesiol Scand 1999;43:563-7. 
3. Ochroch EA, Gottschalk A, Augostides J, Carson KA, Kent L, Malayaman N, et al. Long-term pain and activity during recovery from major thoracotomy using thoracic epidural analgesia. Anesthesiology 2002;97:1234-44.

4. Kehlet H, Jensen TS, Woolf CJ. Persistent postsurgical pain: risk factors and prevention. Lancet 2006;367:1618-25.

5. Blichfeldt-Eckhardt MR, Andersen C, Ørding H, Licht PB, Toft P. From acute to chronic pain after thoracic surgery: the significance of different components of the acute pain response. J Pain Res 2018;11:1541-8.

6. Karmakar MK, Ho AM. Postthoracotomy pain syndrome. Thorac Surg Clin 2004;14:345-52.

7. Macrae WA. Chronic post-surgical pain: 10 years on. Chronic post-surgical pain: 10 years on. Br J Anaesth 2008;101:77-86.

8. Wildgaard K, Ravn J, Kehlet H. Chronic post-thoracotomy pain: a critical review of pathogenic mechanisms and strategies for prevention. Eur J Cardiothorac Surg 2009;36:170-80.

9. Gerner P. Postthoracotomy pain management problems. Anesthesiol Clin 2008;26:355-67.

10. Romero A, Garcia JE, Joshi GP. The state of the art in preventing postthoracotomy pain. Semin Thorac Cardiovasc Surg 2013;25:116-24.

11. Fiorelli A, Izzo AC, Frongillo EM, Del Prete A, Liguori G, Di Costanzo E, et al. Efficacy of wound analgesia for controlling post-thoracotomy pain: a randomized doubleblind study. Eur J Cardiothorac Surg 2016;49:339-47.

12. Imperatori A, Grande A, Castiglioni M, Gasperini L, Faini A, Spampatti S, et al. Chest pain control with kinesiology taping after lobectomy for lung cancer: initial results of a randomized placebo-controlled study. Interact Cardiovasc Thorac Surg 2016;23:223-30.

13. Brockmann R, Klein HM. Pain-diminishing effects of Kinesio $^{\infty}$ taping after median sternotomy. Physiother Theory Pract 2018;34:433-41.

14. Colak MC, Kavakli A, Kilinç A, Rahman A. Postoperative pain and respiratory function in patients treated with electroacupuncture following coronary surgery. Neurosciences (Riyadh) 2010;15:7-10.

15. Sbruzzi G, Silveira SA, Silva DV, Coronel CC, Plentz RD. Transcutaneous electrical nerve stimulation after thoracic surgery: systematic review and meta-analysis of 11 randomized trials. Rev Bras Cir Cardiovasc 2012;27:75-87.

16. Mazzeffi M, Khelemsky Y. Poststernotomy pain: a clinical review. J Cardiothorac Vasc Anesth 2011;25:1163-78.

17. Kase K, Wallis J, Kase J. Clinical Therapeutic Applications of the Kinesio Taping Method. Tokyo: Ken Ikai Co. Ltd; 2003.

18. Krajczy M, Bogacz K, Luniewski J, Szczegielniak J. The influence of Kinesio Taping on the effects of physiotherapy in patients after laparoscopic cholecystectomy. ScientificWorldJournal 2012;2012:948282.

19. Wu WT, Hong CZ, Chou LW. The Kinesio Taping Method for Myofascial Pain Control. Evid Based Complement Alternat Med 2015;2015:950519.

20. Donec V, Kriščiūnas A. The effectiveness of Kinesio Taping $^{\circledR}$ after total knee replacement in early postoperative rehabilitation period. A randomized controlled trial. Eur J Phys Rehabil Med 2014;50:363-71.
21. Montalvo AM, Cara EL, Myer GD. Effect of kinesiology taping on pain in individuals with musculoskeletal injuries: systematic review and meta-analysis. Phys Sportsmed 2014;42:48-57.

22. McCormack HM, Horne DJ, Sheather S. Clinical applications of visual analogue scales: a critical review. Psychol Med 1988;18:1007-19.

23. Hawker GA, Mian S, Kendzerska T, French M. Measures of adult pain: Visual Analog Scale for Pain (VAS Pain), Numeric Rating Scale for Pain (NRS Pain), McGill Pain Questionnaire (MPQ), Short-Form McGill Pain Questionnaire (SF-MPQ), Chronic Pain Grade Scale (CPGS), Short Form-36 Bodily Pain Scale (SF-36 BPS), and Measure of Intermittent and Constant Osteoarthritis Pain (ICOAP). Arthritis Care Res (Hoboken) 2011;63 Suppl 11:S240-52.

24. Bennett M. The LANSS Pain Scale: the Leeds assessment of neuropathic symptoms and signs. Pain 2001;92:14757.

25. Yucel A, Senocak M, Kocasoy Orhan E, Cimen A, Ertas M. Results of the Leeds assessment of neuropathic symptoms and signs pain scale in Turkey: a validation study. J Pain 2004;5:427-32.

26. Melzack R. The short-form McGill Pain Questionnaire. Pain 1987;30:191-7.

27. Yakut Y, Yakut E, Bayar K, Uygur F. Reliability and validity of the Turkish version short-form McGill pain questionnaire in patients with rheumatoid arthritis. Clin Rheumatol 2007;26:1083-7.

28. Hunt SM, McKenna SP, McEwen J, Williams J, Papp E. The Nottingham Health Profile: subjective health status and medical consultations. Soc Sci Med A 1981;15:221-9.

29. Küçükdeveci AA, Kutlay Ş, Gürsel Y. Adaptation of Nottingham Health Profile For Use in Turkey. TThe 8th World Congress of the International Rehabilitation Medicine Association: IRMA 8: August 31 - September 4, 1997, Kyoto (Japan); 1997. p. 373.

30. Fiorelli A, Mazzella A, Passavanti B, Sansone P, Chiodini P, Iannotti $\mathrm{M}$, et al. Is pre-emptive administration of ketamine a significant adjunction to intravenous morphine analgesia for controlling postoperative pain? A randomized, doubleblind, placebo-controlled clinical trial. Interact Cardiovasc Thorac Surg 2015;21:284-90.

31. Katz J, Jackson M, Kavanagh BP, Sandler AN. Acute pain after thoracic surgery predicts long-term post-thoracotomy pain. Clin J Pain 1996;12:50-5.

32. d'Amours RH, Riegler FX, Little AG. Pathogenesis and management of persistent postthoracotomy pain. Chest Surg Clin N Am 1998;8:703-22.

33. Andreae MH, Andreae DA. Regional anaesthesia to prevent chronic pain after surgery: a Cochrane systematic review and meta-analysis. Br J Anaesth 2013;111:711-20.

34. Acar K, Acar H, Demir F, Eti Aslan F. Determining the incidence of postsurgical pain and amount of analgesic use postsurgical pain and analgesic. ACU Sağlık Bil Derg 2016;2:85-9. 